Instituto Internacional de Investigación y Desarrollo Tecnológico Educativo INDTEC, C.A.

DOI: https://doi.org/10.29394/scientific.issn.2542-2987.2017.2.6.10.196-216

OAI-PMH: http://www.indteca.com/ojs/index.php/Revista Scientific/oai

\title{
Uso de las Tic en la Formación Permanente de los Docentes Asesores en la Universidad
}

Autoras: María Virginia Villegas Artigas

Universidad Nacional Experimental "Rafael María Baralt", UNERMB artivillegas@hotmail.com

Trujillo, Venezuela

Roexana Andreina Castillo Vázquez

Universidad de los Andes, ULA

roexanacastillo90@gmail.com

Trujillo, Venezuela

\section{Resumen}

El objetivo del artículo fue proponer el uso didáctico de las Tecnologías de la Información y Comunicación en la formación permanente de los docentes asesores de la Universidad Bolivariana de Venezuela. Se incluyeron enfoques conceptuales de autores como: Ausubel (1963) Gómez, R. (2004), Martínez, S. (2003) entre otros. Este estudio siguió la concepción filosófica positivista, de enfoque cuantitativo, como investigación es proyectiva, con un diseño no experimental, transaccional. El universo objeto de estudio estuvo conformado por 20 docentes. Además, se elaboró una encuesta de tipo cuestionario. Los resultados se presentaron en gráficos y tablas de frecuencias, donde se muestran los porcentajes para cada alternativa de respuesta de acuerdo a la dimensión, indicadores e ítems. Entre las conclusiones se evidenciaron debilidades en cuanto a la aplicación de recursos tecnológicos dentro de la formación permanente de los docentes. Se recomienda establecer planes de capacitación integral para desarrollar las habilidades tecnológicas como uso didáctico en las diferentes áreas del saber universitario.

Palabras clave: tecnologías de información y de comunicación; formación permanente; docente asesor. 


\title{
Use of the Tic in the Permanent Training of Teachers of the University
}

\begin{abstract}
The goal of the investigation research was to propose the didactic use of the Technologies of the Information and Communication in the permanent training of teachers of the Bolivarian University of Venezuela. There were included authors' conceptual approaches as: Ausubel (1963) Gómez, R. (2004), Martínez, S. (2003) among others. This study followed the philosophical positivist conception, of quantitative approach, it's a projective investigation, with a not experimental design, and it is transactional. The universe object of study was formed by 20 teachers. In addition, investigators elaborated a survey of type questionnaire. The results appeared in graphs and tables of frequencies, where the percentages appear for every alternative of response of agreement to the dimension, indicators and articles. On the conclusions, weaknesses were demonstrated as for the application of technological resources inside the permanent training of the teachers. It's recommended to establish plans of integral training to develop the technological skills as didactic use in the different knowledge areas of the university.
\end{abstract}

Keywords: technologies of information and of communication; permanent training; teacher. 


\section{Introducción}

En el contexto de la globalización se tornan necesarios otros escenarios; donde las entidades educativas no pueden ser consideradas como instituciones comisionadas solamente para transmitir los conocimientos a cada generación, sino que tienen una responsabilidad máxima en la formación de un recurso humano que pueda originar los cambios necesarios con el fin de incorporar a cada nación en el marco de los países proactivos, facultados para participar en la competencia. Por lo tanto, una vez que el personal es incorporado a dichas instituciones, rescinde de ser objeto de una atención sistemática con miras a verificar formalmente la praxis académica, el perfil personal, sus capacidades pedagógicas y responsabilidad; por lo que es necesario que se ajusten a patrones previamente descritos para precisar su efectividad en el logro de los objetivos educacionales, considerando, que la función social que este realiza es valorada permanentemente por los estudiantes, profesores, superiores y todas aquellas personas que perciben las acciones que diariamente expresan.

De igual manera, dicho profesional debe poseer una actitud demócrata y socializadora, con convicción de libertad, responsabilidad y respeto hacia los docentes, estudiantes como actores sociales, y propiciar el uso de las Tecnologías de la Información y Comunicación (TIC) como eje integrador que contribuye al desarrollo de potencialidades; razón por el cual se quiere formar al ser social, solidario y productivo, generando espacios de reflexión y la integración en las diferentes áreas del conocimiento, permitiendo el desarrollo de múltiples estrategias pedagógicas para mejorar los procesos de enseñanza y aprendizaje.

De allí el objetivo del presente estudio es Proponer las Tecnologías de la Información y Comunicación en la formación permanente de los docentes, que permita valorar la acción educativa con mayor grado de objetividad e 
imparcialidad posible acerca del mismo, haciendo uso de las (TIC) como componentes para las diferentes áreas de aprendizaje.

\section{Planteamiento y Formulación del Problema}

En la actualidad, los sistemas educativos a nivel mundial, se emprende el desafío de utilizar las Tecnologías de la Información y la Comunicación para dotar a los docentes y estudiantes con las herramientas y los conocimientos precisos que se necesitan en siglo XXI. Por esta razón, los docentes y la enseñanza se encuentran en un mundo de transformación que han dado paso a la implementación de las TIC, a través de los métodos convencionales de enseñanza y aprendizaje, de manera que los docentes y estudiantes tengan acceso al conocimiento y la información.

De igual manera, Palomo, Ruiz y Sánchez, (2006), señalaron que:

Las TIC ofrecen la posibilidad de interacción que pasa de una actitud pasiva por parte del estudiante a una actividad constante, a una búsqueda y replanteamiento continúo de contenidos y procedimientos. Aumenta la implicación del estudiantado en las tareas y desarrollan su iniciativa, ya que se ven obligados constantemente a tomar "pequeñas" decisiones, a filtrar información, a escoger y seleccionar (Palomo, Ruiz y Sánchez, 2006: 56).

De este modo, en América Latina la educación ha dado grandes adelantos en el perfeccionamiento de esquemas pedagógicos, científicos e trasformadores en el abordaje de alternativas de cambio a la práctica educativa, promoviendo una habilidad en el docente con sentido investigativo, generador de nuevos contextos que le permitan fortalecer la educación que se quiere. Venezuela se ha encaminado en el impulso de proyectos y programas que dentro de un consolidado marco jurídico constituyen a las orientaciones orientadas al avance social, financiero y político del país que promueve la democratización y privación social de las TIC por parte de los ciudadanos y 
ciudadanas de la nación, como derechos consagrados en el artículo 110 de la Constitución de la República Bolivariana de Venezuela, donde se reconoce como interés público la ciencia, la tecnología, el conocimiento, la innovación y aplicaciones, de manera que los servicios de información sean vías para lograr el progreso.

En tal sentido, el gobierno venezolano ha fomentado el proyecto Canaima que tiene como fin integrar las TIC a nivel educativo, concretamente en el Subsistema de Educación Media favoreciendo los nuevos modelos educativos que suscita el Estado; donde las TIC s son tomadas como una potencial herramienta para la innovación tanto individual como colectiva de docentes y estudiantes, para brindar la oportunidad para democratizar, emancipar y humanizar la escuela, al mismo tiempo, impulsa la formación holística y el aprendizaje significativo, cuando se aprovechan dentro del espacio de un modelo de educación liberadora y transformadora del ser humano.

Por lo tanto, las tecnologías en el área de la información y la comunicación como herramienta para la formación, le reconoce al docente estar al tanto de manera más directa de los roles que debe asumir dentro de las llamadas nuevas tecnologías de la comunicación, también le permite conocer cómo funcionan estos espacios, contextos donde en un futuro les podría desempeñarse como soporte para seguir desplegando una práctica formativa plenamente en forma virtual.

Reflexionando, desde una realidad donde el docente ya no es la única fuente de información debido que la capacidad para memorizar datos ha dejado de tener en la sociedad, la trayectoria que tenía hace unos años. En consecuencia, las TIC exigen al educador a replantearse desde otra mirada de manera que sea un facilitador que ayude al educando a indagar, elegir y encausar la información derivado de numerosas fuentes. 
Algunos profesores interpretan este cambio como un quebranto del enfoque privilegiado del educador frente al estudiante, donde se descubren confusos ante los esquemas innovadores. En efecto, se proyecta el miedo a un escenario que no puede controlar, el cual lleva a unos docentes a resistir la integración de las TIC en las prácticas pedagógicas; en tal sentido, que los cambios no son irreflexivos ni dependientes de manera exclusiva de la tecnología, sino que pretenden un esfuerzo de conciliación de una representación de labor que consiente cultivar las ventajas que ofrecen. Como Io menciona Martínez, (2003: 78), afirma que "las TIC ayudan al desarrollo de individuos, comunidades, regiones y países, además estas pueden mejorar los niveles educativos e impactar favorablemente en la calidad de vida para acceder a mejores oportunidades para los individuos, la sociedad y la educación".

Así también, en el entorno educativo las exigencias elementales de la educación han ido transformándose; ya que el rápido avance tecnológico hace necesario la incorporación del uso de la computadora, como apoyo a las actividades propias del docente y delos mismos estudiantes. De manera que el uso adecuado de la tecnología, el acceso a internet, el uso de medios audiovisuales, las aplicaciones multimedia además de los juegos educativos, pueden ser importantes para el aprendizaje.

A lo antes expuesto se describe, que el saber está propagado en la sociedad actual, ya que las nuevas tecnologías proporcionan el acceso a un sin número de información, que no ha de confundirse con él. Para que la información devenga en conocimientos el individuo debe apropiársela y reconstruir los conocimientos. Por esta razón, lo primero que debe hacerse explícito es que la incorporación de las nuevas tecnologías en la educación no debe eludir la noción de esfuerzo, por ello, los nuevos recursos informáticos contribuyen al desarrollo del potencial cognitivo de los ciudadanos a través del esfuerzo personal. 
Adicional a esto, las tecnologías componen un medio como jamás ha existido, el cual ofrece un acceso instantáneo a la información, por cuanto esta herramienta constituye un elemento de suma importancia en el proceso educativo, de acuerdo con las necesidades e intereses del nivel de aprendizaje, los parámetros tecnológicos deben ir a la par de la educación, es decir ninguno de ellos puede funcionar de manera aislada.

Por otro lado, estas tecnologías deben estar al servicio del proceso de aprendizaje para contribuir a la formación de los ciudadanos e incluso trabajadores que necesita la sociedad, de manera que tal penetración tecnológica debe estar acompañada de una evolución pedagógica. Siendo así, las nuevas tecnologías exigen un cambio del rol en el profesor-estudiante, donde el docente no puede seguir ejerciendo las funciones tradicionales discursivas a la hora de impartir las clases al estudiante.

Es necesario que los docentes, implemente el uso de las TIC en la fase de aprendizaje, el cual juega un papel importante en la educación y su aplicación de manera adecuada puede ser determinante en el incremento del conocimiento y aprendizaje de los estudiantes e inclusive, permiten generar un aprendizaje constructivista el cual le servirá para toda la vida y no para el momento.

Luego de haber descrito la problemática se formula la siguiente interrogante: ¿Cuál será el uso didáctico de las Tecnologías de la Información y Comunicación en la formación permanente de los docentes asesores?

\section{Marco Teórico}

El presente apartado hace referencia a los antecedentes de la investigación que permite analizar algunas investigaciones que tienen estrecha relación con la variable, bases teóricas que sirven de fundamento a la variable las tecnologías de información y comunicación, asimismo se incluye el sistema de variable. 


\subsection{Tecnología de la Información y Comunicación (TIC)}

Salazar, (2006: 45), en su documento: Orientaciones generales para la incorporación de las Tecnologías de la Información y la Comunicación como eje integrador en el Sistema Educativo Bolivariano, según la Guía del docente, publicada en Enero de (2008: 23). Dados los avances de las TIC, él y la estudiante de hoy, se ven en la necesidad de construir conceptos propios, debido a la diversidad de criterios a los cuales se ven expuestos durante su aprendizaje. Por lo tanto, "él y la docente deberá, optimizar el uso de las herramientas tecnológicas o TIC, en las actividades académicas, con el objetivo de facilitar y estimular en los estudiantes el placer por el aprendizaje". Además, Miratía (2005: 16), refiere que "el director y los docentes han de asumir el papel de mediador u orientador en ese proceso de construcción de significados".

Para Cabero (2000: 19), las TIC, son "medios e instrumentos técnicos, tales como: video interactivo, videotexto y teletexto, televisión por satélite y cable, hiperdocumentos, CD-ROM en diferentes formatos, sistemas multimedia, tele y videoconferencia, los sistemas expertos, correo electrónico, telemática, virtual y de servicios". García (1996: 12), señala que las TIC "se refieren fundamentalmente a tres grandes sistemas de comunicación: el video, la informática y la telecomunicación".

\subsection{Formación del Docentes en el Uso de las Tecnologías de la Información y Comunicación (TIC).}

La actualización es la acción de adquirir nuevas competencias, es decir, de adecuarse y actualizar los conocimientos y habilidades. Autores como Paredes, Murillo y Egido (2005: 25), definen "la actualización profesional como el conjunto de políticas, estrategias y procesos que están orientados a formar y capacitar de manera continua y permanente a la fuerza laboral". El autor anteriormente citado, hace referencia sobre el tema expuesto estableciendo 
que la formación profesional es un derecho reconocido en la normativa laboral, de carácter internacional y como se encuentra condicionado al goce del derecho y trabajo desplegado por los docentes.

A tal efecto Stonner y otros (1996: 417), señalan: Que "la capacitación se propone mantener y mejorar el desempeño actual en el trabajo". Otro autor como Hargreeves y Fullan, citado por Torres (2006: 67), dice que "el desarrollo profesional docente, implica suministrar a éste de conocimientos en concordancia con el área en el cual se desempeña, el desarrollo personal y el contexto educativo". Por lo que, la formación debe dirigir la acción a modificar aptitudes, elevar el nivel de eficiencia y bienestar personal. Debe ser una actividad eminentemente práctica, humanista, innovadora que redunde en el incremento de la calidad del desempeño en el trabajo docente.

Desde esta perspectiva, la preparación constituye un recurso estratégico que debe implementar con urgencia, debe contar con un conjunto de acciones como; jornadas, talleres de sensibilización y cursos de actualización, que conduzcan a mejorar la labor docente, para que puedan brindar, compartir conocimientos con la finalidad de despertar en el estudiante las potencialidades así obtener lo mejor de él y ejecútese a proyectos y buscar soluciones rápidas y efectivas a cualquier problema relacionado con ella.

\subsection{Impacto de la Sociedad de la Información en el Mundo Educativo.}

Esta emergente sociedad de la información, impulsada por un vertiginoso avance científico en un marco socioeconómico neoliberalglobalizador y fundamentada por el uso general de las potentes y diversas tecnologías de la información y la comunicación (TIC), conlleva cambios que alcanzan todos los ámbitos de la actividad humana. Aviram (2002: 38), identifica "tres posibles modificaciones de los centros docentes para familiarizarse a las TIC y al nuevo contexto cultural". 
3.4. Escenario Tecnócrata: se realizan en las instituciones donde se establecen algunos ajustes: en primer lugar, la introducción de la "alfabetización digital" de los estudiantes en el curriculum, donde deben aprender sobre las TIC y seguidamente implementaran de forma progresiva la utilización de las TIC como instrumento productivo para el proceso de la información, fuente de información y proveedor de materiales didácticos (aprender de las TIC).

3.5. Escenario Reformista: a través de este contexto, se proporcionan la integración de los tres niveles que conforman las TIC que sugieren Martín, Beltrán y Pérez (2003): "Ios dos primeros (aprender sobre las TIC y aprender de las TIC) y por otro lado, se introducen en las prácticas docentes nuevos métodos de enseñanza/aprendizaje constructivistas que contemplan el uso de las TIC como instrumento cognitivo (aprender con las TIC) y para la realización de actividades interdisciplinarias y colaborativas".

3.6. Escenario Holístico: estos centros realizan una intensa reestructuración de todos sus elementos. Como indica Majó (2003):

La escuela y el sistema educativo no solamente tienen que enseñar las nuevas tecnologías, no sólo tienen que seguir enseñando materias a través de las nuevas tecnologías, sino que estas nuevas tecnologías aparte de producir unos cambios en la escuela producen un cambio en el entorno y, como la escuela lo que pretende es preparar a la gente para este entorno, si éste cambia, la actividad de la escuela tiene que cambiar (Majó, 2003: 72).

Por lo que, los efectos se manifiestan de forma muy particular en las actividades laborales y en el ámbito educativo, donde todo debe ser analizado, comenzando por el origen de la escuela y las demás instituciones educativas, hasta la formación básica que precisan a las personas la forma de enseñar y 
de aprender, los métodos que utilizan para ello, además de la estructura organizativa de las instituciones y su cultura.

\subsection{Las Tecnologías de la información y la comunicación operadas por los docentes.}

La "sociedad de la información" en general y las recientes tecnologías, inciden de forma significativa en todos los niveles del mundo educativo. Las actuales generaciones van alcanzando de manera natural esta nueva cultura que va conformando y que conlleva muchas veces a importantes esfuerzos de formación, adaptación y "desaprender" muchos elementos que ahora "se realizan de otra manera" o que simplemente ya no se utilizan. Los más jóvenes no tienen el paso experiencial de haber convivido en una sociedad "más estática”, de manera que para este grupo la innovación y el aprendizaje a continuado con el fin de conocer los cambios que se van presentando continuamente (Majó, 2003: 84).

Es cierto que para favorecer el proceso que se comienza a desarrollar desde los ambientes educativos informales (familia y ocio), la institución debe integrar también la nueva cultura: alfabetización digital, fuente de información, elemento productivo para realizar actividades, material didáctico e instrumento cognitivo.

Además del uso y disfrute de los medios tecnológicos (en clase y en casa), permitirá realizar actividades educativas dirigidas a el desarrollo psicomotor, cognitivo, emocional y social, las actuales tecnologías también pueden favorecer a aumentar el contacto con las familias.

\subsection{Formación Permanente del Docente.}

La formación permanente debe asumirse como un proceso que promueve la reflexión colectiva sobre la acción pedagógica para enriquecerlo y producir conocimiento didáctico, que atenúe la confrontación con otros 
puntos de vistas, promueve el análisis de nuevos conocimientos pedagógicos y estimula la investigación sobre el que hacer educativo. Dentro de este marco de ideas, Trosino (2000: 26), considera a la formación como "un proceso de obtención de conocimientos y desarrollo de aptitudes, que permiten la preparación integral del hombre para una vida activa, productiva y satisfactoria, así como para un eficiente desempeño en cualquier nivel de calificación y responsabilidad".

Partiendo lo señalado por el autor, se puede expresar que la formación firme del educador es un mecanismo clave en el transcurso de renovación e innovación didáctica encaminada al avance de la calidad de la enseñanza, desde este punto de vista, la acción debe concebirse con los educadores al igual que realizando investigaciones educativas con ellos.

Por lo tanto, la formación es el proceso que desarrolla el aprendizaje y facilita un contexto para el mismo en tres terrenos principales. El primero es el conocimiento y como emplearlo, el segundo es el aprendizaje de experiencias y el tercero es el aprendizaje de valores. En ese orden de ideas, Díaz y Hernández, (2002: 31), señalan:

La formación profesional involucra el estudio de los fenómenos educativos de la docencia planteándose múltiples aproximaciones disciplinarias, dada la complejidad que representa no solo la explicación de los procesos de aprendizaje y desarrollo personal involucrados, como por la necesidad de disponer tanto de un marco de referencias específicas, facilitando orientar la práctica (Díaz y Hernández, 2002: 31).

Es importante señalar, la acción educativa se ejerce en un marco y en un medio que las más de las veces predeterminan, limitan o inhiben, potencian o estimulan el sentido o la disposición que los educadores pretenden imprimir a su quehacer pedagógico; la instituciones requieren de una dirección y de un liderazgo preparados para dinamizar efectivamente ese quehacer, de 
estimular los cambios de gestionarlos adecuadamente y de provocar, articular los procesos de participación efectiva de sus miembros.

\section{Marco Metodológico}

Toda investigación requiere ampliar los hechos y relaciones. Por consiguiente, los resultados o nuevos conocimientos que se obtuvieron tienen el mayor grado de exactitud y confiabilidad, por lo tanto, se hizo necesario establecer una metodología o procedimiento ordenado para construir el significado de hechos y fenómenos hacia donde estuvieron encaminados los intereses de la investigación. En tal sentido, la metodología es un proceso científico a través del cual se alcanzaron los objetivos planteados, esto permitió la estructuración sistemática metodológica para lograr la interpretación de los resultados en función del problema y los planteamientos teóricos presentados.

Por tal motivo, la presente investigación es de carácter descriptiva, ya que se obtuvo información real donde se identificaron los diferentes elementos, que permitieron saber quién, dónde, cuándo, cómo y el porqué del problema planteado, el cual es uso didáctico de las TIC en la formación permanente de los docentes de la universidad Bolivariana de Venezuela

Este estudio corresponde a un diseño no experimental, transeccional o transversal de campo. Según Hernández y otros (2006: 205), "la investigación de tipo no experimental, se realiza sin manipular deliberadamente las variables, los fenómenos se observarán tal y como se presenta en su contexto natural para después analizarlos". Se puede decir que en esta investigación no experimental no es posible manipular la variable o asignar aleatoriamente a los sujetos o los tratamientos, esto debido a que no se constituye ninguna situación, sino que se perciben situaciones ya existentes, no suscitadas intencionalmente en el estudio. 
Para Tamayo y Tamayo (2001: 114), "la población está determinada por la totalidad del fenómeno a estudiar donde las unidades de población poseen una característica común la cual se estudia y proporciona origen a los datos que se necesitan para la investigación". Del mismo modo, Chávez (2007: 162), "es el universo de la investigación, sobre el cual se pretende generalizar los resultados y está constituida por características o estratos que le permiten distinguir los sujetos, unos de otros". Estas características de la población se deben delimitar con la finalidad de establecer los parámetros muestrales, donde se incluye en esta totalidad de los sujetos, objetos, fenómenos o situaciones que se desea investigar.

Por otro lado, Silva (2006: 96), señala que es "la totalidad del fenómeno a estudiar, cuyas unidades de análisis poseen características comunes, las cuales se estudian y proporcionan origen a los fundamentos de la investigación". En este sentido, la población de estudio quedó constituida por 20 sujetos, debido a que es solo el personal docente de la Universidad Bolivariana de nuevas tecnologías en la formación permanente de los docentes asesores de la Universidad Bolivariana de Venezuela, Fundación Misión Sucre, Municipio Sucre, Estado Zulia, de Venezuela.

5. Cuadro. Resultados.

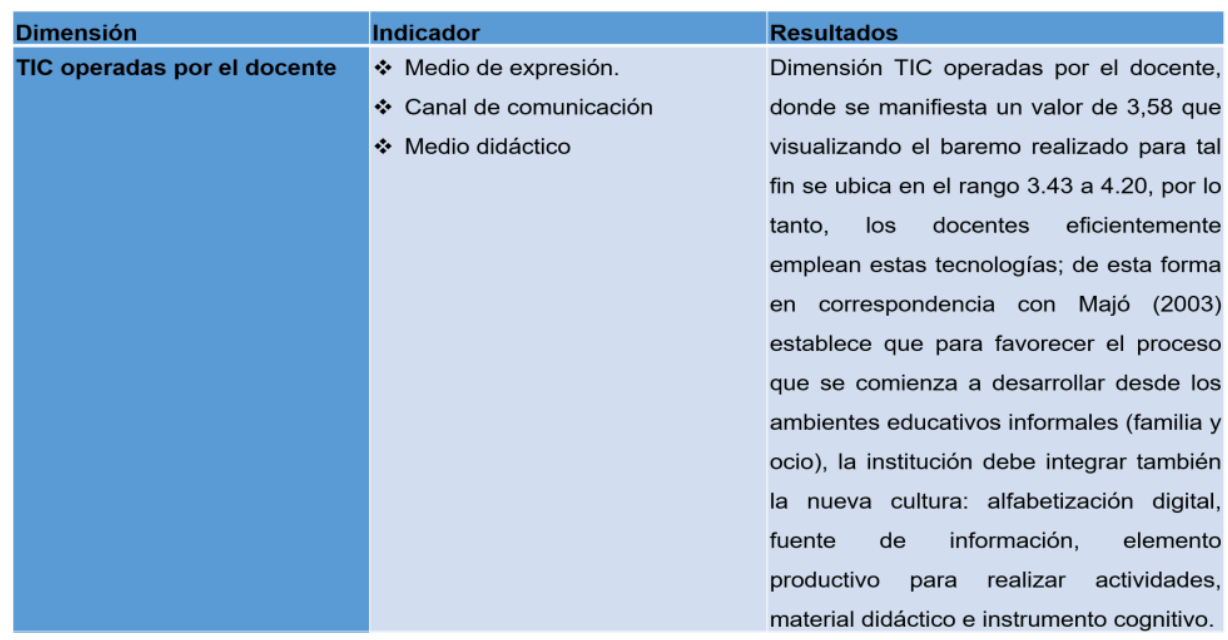




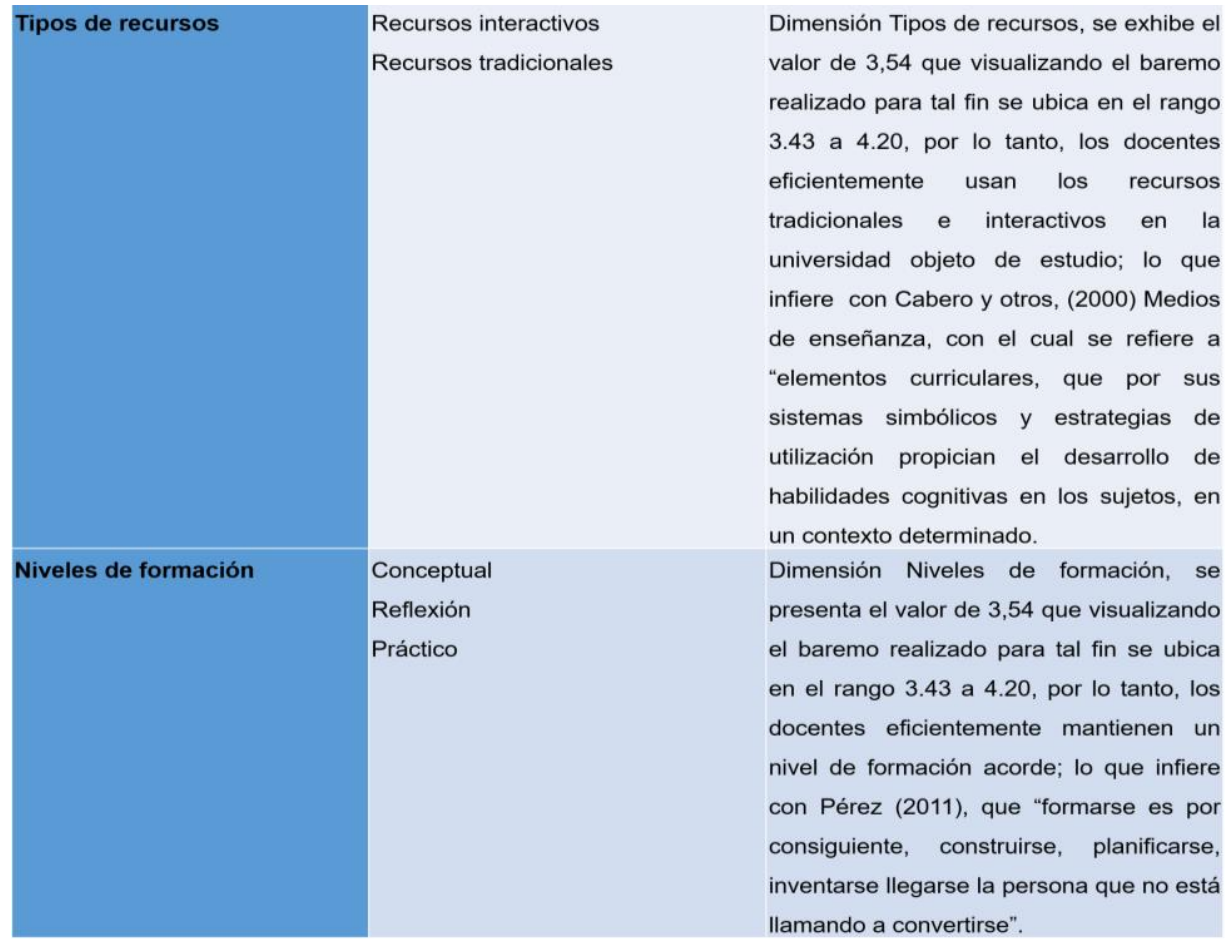

Fuente: (2017) Terán, Vázquez y Villegas.

\section{Conclusiones}

Con respecto a las reflexiones finales los docentes disponen de recursos tanto interactivos como tradicionales para el desarrollo de las actividades diarias del aula; por otro lado, llama la atención que existe algunos docentes que poco toman en cuenta los recursos interactivo por tanto es de pensar que se enfocan más en lo tradicional; de esta forma es necesario aplicar estrategias para que se actualicen en los diferentes uso que proporcionan las tecnologías, por lo tanto se deduce que existe niveles de formación pedagógica acordes con lo planteado por la institución educativa, sin embargo existe fallas donde es necesario aplicar herramientas de capacitación de manera práctica y de contenido de esta forma minimizar estas situación, ya que un pequeño porcentaje necesita ser orientado con respecto a las tecnologías de innovación. 


\section{Propuesta}

Con base a los resultados derivados de la investigación, se establecen el diseño de una propuesta para el uso didáctico de las tecnologías de la información y la comunicación en la formación permanente de los docentes asesores de la Universidad Bolivariana de Venezuela.

\subsection{Aplicación de las tecnologías dentro de la educación}

Herramientas tecnológicas que se pueden trabajar desde la educación. a). Presentaciones:

Son herramientas tecnológicas muy adecuadas para presentar los aspectos teóricos de cualquier asignatura, así como cualquier unidad didáctica que se quiera que los estudiantes conozcan a nivel teórico, previo a la realización práctica. Son muchos los soportes para realizar las presentaciones: POWER POINT, OPEN OFFICE IMPRESS, entre otros.

Experiencia en el aula: esta herramienta se puede utilizar a principio de una unidad didáctica, para que los estudiantes tengan conocimientos previos del trabajo en la unidad. En la web existen páginas de internet que ofrecen presentaciones de diferentes contenidos las cuales se pueden utilizar como ejercicio dentro de la clase, para luego interactuar con aspectos del tema.

b). WebQuest:

Una WebQuest es un tipo de actividad didáctica que consiste en una investigación guiada, con recursos principalmente procedentes de Internet, que promueve la utilización de habilidades cognitivas superiores, el trabajo cooperativo y la autonomía de los estudiantes e incluye una evaluación. Las WebQuest son utilizadas como recurso didáctico por los profesores, puesto que permiten el desarrollo de habilidades de manejo de información y el desarrollo de competencias relacionadas con la sociedad de la información. 
Experiencia en el aula: esta acción educativa es muy adecuada para reforzar ciertos aprendizajes y mejorar otros relacionados con la competencia digital ya que es el estudiante el que debe buscar información en internet sobre el tema propuesto. Se proponen contenidos referidos "al cuerpo humano" o "actividades motrices" entre otros, así se aprenden diferentes tipos de calentamientos en función de las actividades que posteriormente van a trabajar, ya que se pretende conseguir una mayor autonomía en el mismo.

\section{c). Los Blogs:}

Un blog, o en español también una bitácora, es un sitio Web periódicamente actualizado que recopila cronológicamente textos o artículos de uno o varios autores, apareciendo primero el más reciente, donde el autor conserva siempre la libertad de dejar publicado lo que crea pertinente. El nombre bitácora está basado en los cuadernos de bitácora, cuadernos de viaje que se utilizaban en los barcos para relatar el desarrollo del viaje y que se guardaban en la bitácora. Aunque el nombre se ha popularizado en los últimos años a raíz de su utilización en diferentes ámbitos, el cuaderno de trabajo o bitácora ha sido utilizado desde siempre.

Experiencias en el aula: Dentro de una asignatura es una herramienta tecnológica que se puede utilizar con frecuencia, fundamentalmente al colocar enlaces sobre actividades JClic que los estudiantes trabajarían. El blog permite organizar todo aquello que queremos que los estudiantes trabajen, a través de enlaces, directamente a aquellos sitios Web para facilitar su acceso y búsqueda a la información de la unidad correspondiente.

Durante el transcurso de esta propuesta se mencionó diferentes herramientas tecnológicas que se pueden utilizar dentro de las asignaturas, así del momento que se ha considerado más adecuado para llevarlas a la práctica. Pero también es cierto, que será cada docente, en última instancia, el que considere qué momento es el más oportuno, en función del contexto 
donde desarrolla su programación anual, de las instalaciones, materiales disponibles e incluso del tiempo atmosférico que lo acompañe durante el transcurso del año escolar.

También es importante destacar las grandes posibilidades que ofrecen las tecnologías para el desarrollo de cualquier asignatura, como contribuir a la mejora integral del estudiante, así se fomentara sus otros planos como son los intelectuales y los sociales, ambos perfectamente vinculantes con el trabajo y el desarrollo de la competencia digital.

\section{Referencias}

Aviram, A. (2002). "ICT in education: should it necessarily be a case of the recurrent reinvention of the Wheel?". London: Kluwer (forthcoming). Cabero, J. (2000). Las Nuevas Tecnologías de la Información y la Comunicación: aportaciones a la enseñanza. Madrid.

Chávez, N. (2007). Metodología de la Investigación Educativa. Zulia.

Constitución de la República Bolivariana de Venezuela. (1999). Caracas, Venezuela.

Díaz, F. y Hernández, G. (2002). Estrategias Docentes para un Aprendizaje Significativo McGraw - Hill. Universidad Nacional Autónoma de México, México.

García, A. (1996). Las nuevas tecnologías en la formación del profesorado. España: Narcea, S.A. de Ediciones. pp. 185-199.

Hernández, R. Fernández, C. y Baptista, P. (2006). Metodología de la Investigación. Editorial McGraw-Hill Interamericana. México.

Majó, J. (2003). Nuevas Tecnologías y Educación. Recuperado de: http://www.uoc.edu/web/esp/articles/joan majo.html

Martín Patiño, José María; Beltran Llera, Jesús; Pérez, Luz (2003). Cómo aprender con Internet. Madrid: Fundación Encuentro. 
Martínez, S. (2003). "La Calidad en el Proceso de Formación Vía Internet: El Planteamiento de la Universidad Politécnica de Valencia". III Congreso Aplicación de las Nuevas Tecnologías en la Docencia.

Miratia (2005). La Tecnologías de la Información y la Comunicación en la Educación. Infobit: Venezuela: Ministerio de Educación y Deporte.

Palomo, Ruiz y Sánchez (2006). TIC's como Instrumento Indispensable en Ios Centros Educativos. Editorial Altablero. Presencial y e-learning, Valencia.

Paredes, J., Murillo, J. y Egido, I. (2005). El profesor ante la innovación y el cambio educativos. Análisis de las actitudes. Madrid: Dpto. de Didáctica y Teoría de la Educación

Salazar, L. (2006). Los Servicios de Internet Como Recurso Didáctico en la Elaboración de Proyectos Pedagógicos en la Educación Bolivariana. Trabajo especial de grado. Universidad Santa María.

Silva, J. (2006). Metodología de la Investigación. Ediciones COBO, Venezuela.

Stoner, J. y Freeman, E. y Gilbert, D. (1996). Administración. Editorial Pantice Hall Hispanoamericana S. A. 6ta Edición. México.

Tamayo y Tamayo (2001). El Proceso de la Investigación Científica. Editorial LIMUSA, S.A. México.

Torres, S., J. (2006). La desmotivación del profesorado. Madrid: Morata. Trosino, C. (2000). El ABC del Instructor. México. 


\section{María Virginia Villegas Artigas \\ e-mail: artivillegas@hotmail.com}

Nacida en Valera Edo Trujillo, Venezuela. Egresada de

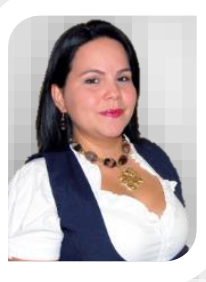
la Universidad Nacional Experimental Simón Rodríguez, con el Titulo de Lcda. En Educación Integral sin Mención. Obtuvo el título de M.Sc. en Educación Superior de la Universidad Rafael María Baralt, en esta misma casa de estudio culminó la escolaridad para optar por el título de Doctora en Educación. Con 8 años de servicio, comenzando como Docente de aula en la U.E. Colegio San José en el año escolar 2008-2009, actualmente, es la Coordinadora Académica de dicha Institución. 
Nacida en Valera, estado Trujillo, Venezuela.

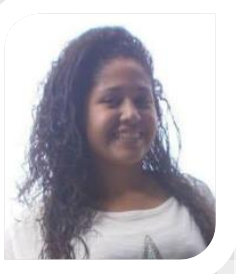

Estudiante de educación mención Lenguas Extranjeras del Núcleo Universitario "Rafael Rangel" ULA. Fue preparadora en el área de francés por dos (2) años. Ha participado en numerosos eventos de investigación en distintas áreas como ponente, comité organizador y asistente. Adscrita al Centro de Investigaciones Lingüísticas y Literarias "Mario Briceño Iragorry" ULA-NURR. Miembro de la línea de investigación Memoria, Educación y Discursos emergentes (UNERMB). Miembro del Taller "AbyaYala" NURR.

El contenido de este manuscrito se difunde bajo una Licencia de Creative Commons ReconocimientoNoComercial-Compartirlgual 4.0 Internacional 